\title{
98. Certain Congruences and the Structure of Some Special Bands
}

\author{
By Miyuki YaMADA \\ Shimane University \\ (Comm. by K. KunUGi, M.J.A., July 12, 1960)
}

1. A band is synonymous with an idempotent semigroup. Let $S$ be a band, and $S \sim \Sigma\left\{S_{r}: \gamma \in \Gamma\right\}$ its structure decomposition (cf. Kimura [1]). For each subset $\Delta$ of $\Gamma$, we first define the relation $\Re_{4}$ on $S$ as follows:

$a \Re_{\Delta} b$ if and only if $\left\{\begin{array}{c}a b=a \text { and both } a \text { and } b \text { are contained in } \\ \text { the same } S_{r}, \gamma \in \Delta, \\ \text { or } \\ a b=b \text { and both } a \text { and } b \text { are contained in } \\ \text { the same } S_{r}, \gamma \notin \Delta .\end{array}\right.$

Then, it is easily seen that $\Re_{\Delta}$ is an equivalence relation on $S$ but not necessarily a congruence.

The following two theorems have been proved by Kimura [2]:

Theorem I. $\Re_{\phi}\left(\Re_{\Gamma}\right)$, where $\phi$ is the empty subset of $\Gamma$, is a congruence on $S$ if and only if $S$ is left (right) semiregular. Further, in this case the quotient semigroup $S / \Re_{\phi}\left(S / \Re_{\Gamma}\right)$ is left (right) regular.

Theorem II. Both $\Re_{\phi}$ and $\Re_{\Gamma}$ are congruences on $S$ if and only if $S$ is regular. Further, in this case $S$ is isomorphic to the spined product of $S / \Re_{\phi}$ and $S / \Re_{\Gamma}$ with respect to $\Gamma$.

In this note, we shall present a necessary and sufficient condition for $\Re_{\Delta}$ to be a congruence on $S$, and make some generalizations of Theorems I and II. However here only the main results and necessary definitions are given, and the proofs are all omitted. We will study them in detail elsewhere. ${ }^{1)}$

Notations and terminologies. If $M$ and $N$ are two sets such that $M \supset N$, then $M \backslash N$ will denote the complement of $N$ in $M$. The notation $\phi$ will denote always the empty set. Throughout the whole paper $S$ will denote a band, unless otherwise mentioned. The structure semilattice of $S$ and the $\gamma$-kernel, ${ }^{2)}$ for each $\gamma$ of the structure semilattice, will be denoted by $\Gamma$ and $S_{r}$ respectively. And the structure decomposition of $S$ will be denoted naturally by $S \sim \Sigma\left\{S_{r}: \gamma \in \Gamma\right\}$. Any other notation or terminology without definition should be referred to $[1]$.

2. Let $\Delta$ be a subset of the structure semilattice $\Gamma$ of $S$, and

1) This is an abstract of the paper which will appear elsewhere.

2) For definition, see [1]. 
put $\bigcup_{\delta \in \Delta} S_{\delta}=S(\Delta)$. First of all, we shall define here $(\Gamma, \Delta)$-semiregularity, $\Gamma(\Delta)$-regularity and quasi-regularity.

$S$ is called $(\Gamma, \Delta)$-semiregular or $\Gamma(\Delta)$-regular if it has the following corresponding property $(\mathrm{P})$ or $\left(\mathrm{P}^{*}\right)$.

$$
\begin{cases}c a b a c b a=c a b a & \text { if } a b \in S(\Delta) \text { and } a b c \in S(\Delta) . \\ a b a c=b a c & \text { if } a b \in S(\Delta) \text { and } a b c \notin S(\Delta) . \\ c a b a=c a b & \text { if } a b \notin S(\Delta) \text { and } a b c \in S(\Delta) . \\ a b c a b a c=a b a c & \text { if } a b \notin S(\Delta) \text { and } a b c \notin S(\Delta) .\end{cases}
$$

$$
\left\{\begin{array}{l}
\begin{array}{l}
c a b a c b a=c a b a \\
a b c a b a c=a b a c
\end{array} \\
\left.\begin{array}{l}
a b a=c a b \\
a b a c=b a c
\end{array}\right\} \quad \begin{array}{c}
\text { if } a b \in S(\Delta) \text { and } a b c \in S(\Delta), \text { or if } a b \notin S(\Delta) \\
\text { and } a b c \notin S(\Delta) .
\end{array} \quad \text { and } a b c \in S(\Delta) \text { and } a b c \notin S(\Delta), \text { or if } a b \notin S(\Delta)
\end{array}\right.
$$

Further, $S$ is called quasi-regular if it becomes $\Gamma(\Delta)$-regular for some subset $\Delta$ of $\Gamma$.

Of course, it is clear from the definition that for an arbitrary $\Gamma_{1} \subset \Gamma, \Gamma\left(\Gamma_{1}\right)$-regularity is equivalent to $\Gamma\left(\Gamma \backslash \Gamma_{1}\right)$-regularity.

Under these definitions, we have

Lemma 1 . $S$ is $\Gamma(\Delta)$-regular if and only if it is both $(\Gamma, \Delta)$ and $(\Gamma, \Gamma \backslash \Delta)$-semiregular.

Lemma 2. $S$ is quasi-regular if and only if it is the class sum of two subsets $A, B$ such that:

(1) If $A \ni a, a x a=a$ and $x a x=x$, then $x \in A$.

(2) If $B \ni b, b y b=b$ and $y b y=y$, then $y \in B$.

(3) If $A$ э $a b$ and $A \ni a b c$, , then cabacba=caba and abcabac $\left\{\begin{array}{l}\text { or } \\ B \ni a b \text { and } B \ni a b c\end{array}\right\}=a b a c$.

(4) If $\left\{\begin{array}{l}A \ni a b \text { and } B \ni a b c, \\ o r \\ A \ni a b c \text { and } B \ni a b\end{array}\right\}$, then $a b a c=b a c$ and $c a b a=c a b$.

Next, we shall define bi-regularity of bands: $A$ band $G$ is called bi-regular if for any given elements $a, b$. of $G$ it satisfies at least one of the relations $a b a=b a$ and $a b a=a b$.

The global structure of bi-regular bands is given by

Theorem 1. $S$ is bi-regular if and only if each $\gamma$-kernel is left or right singular.

Let $G \sim \Sigma\left\{G_{\omega}: \omega \in \Omega\right\}$ be the structure decomposition of a bi-regular band $G$. From Theorem 1, every $\omega$-kernel is then left or right singular. Let $\Lambda$ be a subset of $\Omega$.

$G$ is said to be $(\Omega, \Lambda)$-regular if it satisfies the following (C):

$\left\{\begin{array}{l}\text { For } \alpha \in \Lambda, G_{\alpha} \text { is left singular. } \\ \text { For } \beta \notin \Lambda, G_{\beta} \text { is right singular. }\end{array}\right.$

It is sometimes possible that $G$ is both $\left(\Omega, \Lambda_{1}\right)$ - and $\left(\Omega, \Lambda_{2}\right)$-regular 
for some different subsets $\Lambda_{1}$ and $\Lambda_{2}$. Let $G_{1}$ and $G_{2}$ be bi-regular bands having the same $\Omega$ as their structure semilattices. Let $G_{1} \sim \Sigma\left\{G_{\omega}^{1}: \omega \in \Omega\right\}$ and $G_{2} \sim \Sigma\left\{G_{\omega}^{2}: \omega \in \Omega\right\}$ be their structure decompositions.

Then, $G_{1}$ and $G_{2}$ are called mutually associated bi-regular bands if

for any given $\omega \in \Omega\left\{\begin{array}{l}G_{\omega}^{1} \text { is left singular and } G_{\omega}^{2} \text { is right singular, } \\ \text { or } \\ G_{\omega}^{1} \text { is right singular and } G_{\omega}^{2} \text { is left singular. }\end{array}\right.$

3. The next two theorems are generalizations of Theorems I and II.

Theorem 2. $\Re_{\Delta}$ is a congruence on $S$ if and only if $S$ is $(\Gamma, \Delta)$ semiregular. Further, in this case the quotient semigroup $S / \Re_{\Delta}$ is a $\left(\Gamma, I^{\top} \backslash \Delta\right)$-regular band, having $S / \Re_{4} \sim \Sigma\left\{S_{\gamma} / \Re_{4}: \gamma \in \Gamma\right\}$ as its structure decomposition.

Theorem 3. Both $\Re_{\Delta}$ and $\Re_{\Gamma \backslash \Delta}$ are congruences on $S$ if and only if $S$ is $\Gamma(\Delta)$-regular. Further, in this case $S$ is isomorphic to the spined product of $S / \Re_{4}$ and $S / \Re_{\Gamma \backslash 4}$ with respect to $\Gamma$.

Combining Lemmas 1 and 2 with Theorems 2 and 3, we obtain the following corollaries.

Corollary. If $S$ is $\Gamma(\Delta)$-regular, then it is isomorphic to the spined product of a $(\Gamma, \Gamma \backslash \Delta)$-regular band and a $(\Gamma, \Delta)$-regular band with respect to $\Gamma$.

Corollary. If $S$ is quasi-regular, then it is isomorphic to the spined product of mutually associated bi-regular bands with respect to $\Gamma$.

Corollary. If $S$ can be decomposed into the class sum of two subsets $A, B$ having the properties (1)-(4) in Lemma 2, then it is isomorphic to the spined product of mutually associated bi-regular bands with respect to $\Gamma$.

Remark. The existence of a band which is quasi-regular but neither left semiregular nor right semiregular can be verified by giving an example.

\section{References}

[1] N. Kimura: Note on idempotent semigroups. I, Proc. Japan Acad., 33, 642645 (1957).

[2] N. Kimura: Ditto. III, Proc. Japan Acad., 34, 113-114 (1958). 